\title{
On voltage-current characteristics and critical current in Bi-2212
}

\author{
A. Pautrat, ${ }^{1, *}$ Ch. Simon,${ }^{1}$ J. Scola,${ }^{1}$ C. Goupil,${ }^{1}$ A. Ruyter,${ }^{2}$ L. Ammor, ${ }^{2}$ P. Thopart,${ }^{3}$ and D. Plessis ${ }^{3}$ \\ ${ }^{1}$ CRISMAT/ENSI-Caen, UMR 6508 du CNRS,6 Bd Marechal Juin, 14050 Caen, France. \\ ${ }^{2}$ LEMA-Université de Tours UMR CNRS/CEA 6157 - 37000 TOURS France. \\ ${ }^{3}$ CEA/DMAT B.P. 16 Le Ripault - 37260 MONTS France.
}

\begin{abstract}
$\mathrm{V}(\mathrm{I})$ characteristics have been performed in a monocrystalline microbridge of $\mathrm{Bi}[\mathrm{Pb}]-2212$. The vortex phase diagram has been greatly investigated. Linear but non-ohmic Voltage(Current) (V(I)) curves with well defined critical current have been observed. A departure from this behavior is observed near the peak effect where an out of equilibrium high threshold current can be stabilized. At high temperature, the critical current persists in the "liquid" state despite the dissipation at the lowest bias. Some implications of these results are discussed. In particular, it is proposed that the surface disorder, rather than the bulk disorder, is responsible for the vortex pinning in this sample.
\end{abstract}

PACS numbers: 74.25.Sv,74.25.Fy,74.72.Hs

The generic vortex phase diagram in cuprates is now described in terms of into different thermodynamic phases of vortex [1]. The main idea is that an ordered Vortex Lattice (VL) is present at low field and low temperature, and that it develops into a phase possessing a degraded order when the thermal fluctuations or the static disorder are increased. This corresponds respectively to (ordered solid/liquid) and (ordered solid/disordered solid) transitions. The strong experimental facts which have justified these ideas are the disappearance of the vortex pinning in the "liquid" phase and the increase of this vortex pinning (the peak effect) when crossing the disordering transition. The highly anisotropic cuprate $\mathrm{Bi}-2212$ can be taken as a representative sample where these three states can appear. Nevertheless, recent experiments suggest to qualify this point of view. Indeed, it has been found that both the high field and the high temperature properties can be interpreted with the same state of vortex matter, meaning that no difference between them should be presupposed [2, 3]. Another striking result is that VL translational order is not a good order parameter to characterize the first order transition [4], whereas one could have expected the contrary for a genuine melting. From an experimental point of view, the VL behavior in $\mathrm{Bi}-2212$ has been tackled at low field by magneto-optic imaging or local ac probes [2, 3], which are sensitive to pinning induced screening currents on the surface of the samples. If the magnetic field is increased, the limited resolution of the above-cited experiments strongly restricts their ability to give information on the VL pinning and dynamical properties. One complementary and easily understandable experiment is the measure of a voltage versus current $(\mathrm{V}(\mathrm{I}))$ curve. The critical current $I_{c}$, which gives the pinning ability of the medium, can be extracted without the need of complicated and possibly unjustified assumptions. Furthermore, it has been shown by numerous theories and simulations [5, 6, 7] that the nature of the VL

*corresponding author: alain.pautrat@ensicaen.fr should govern the variation of its velocity as function of a bulk training force. To the extent that this force is directly given by the amount of applied transport current, these signatures are directly expected in the $\mathrm{V}(\mathrm{I})$ curves. For example, there is some consensus concerning the dynamical properties of a VL [1]. They are expected to be dominated by hopping over barriers at low currents, with a transition to a free flow and Ohmic-like regime at high current. Fig.1 is a schematic representation of the different $\mathrm{V}(\mathrm{I})$ curves which can be expected for the different VL states, namely ordered state (Bragg Glass) and disordered states (vortex Glass and vortex liquid). We note that if numerical simulations of velocity versus force curves are extremely numerous in the literature, very few direct measurements have been performed in cuprates to see if the main expectations compare well with the experiments. In the present experiment, we are thus principally interested in the qualitative differences (if any) between the functional form of the $\mathrm{V}(\mathrm{I})$ curves, for different locations $(B, T)$ in the phase diagram.

The measurement of a V(I) curve is conceptually a very simple method and is currently used as a useful probe of vortex properties in low $\mathrm{T}_{c}$ materials. On the contrary, it was unemployed for high $\mathrm{T}_{c}$ 's, except using very low current densities. The reason is practical: quasi-perfect contacts are usual in low $\mathrm{T}_{c}$ materials $\left(R_{\text {contacts }} \lesssim 0.001 \Omega\right)$ but not in cuprates $\left(R_{\text {contacts }} \gtrsim 1-2 \Omega\right)$. It is thus difficult to avoid any overheating in these rather resistive contacts as soon as the current exceeds tens of milliAmperes. Typically, under normal Helium atmosphere and standard experimental conditions, I $\approx 100 \mathrm{~mA}$ into a $1 \Omega$ metallic contact $\left(\lesssim \mathrm{mm}^{2}\right)$ is compatible with a local increase of the temperature of about $0.1 \mathrm{~K}$. This strongly restricts the value of the "safe" transport current. Extrapolating to a typical sample size $(\mathrm{W}=1 \mathrm{~mm}, \mathrm{t}=100 \mu \mathrm{m}, \mathrm{W}=$ width, $\mathrm{t}=$ thickness), the $\mathrm{V}(\mathrm{I})$ curves are thus difficult to perform as soon as $J \gtrsim 10^{2} \mathrm{~A} / \mathrm{cm}^{2}(i \gtrsim 0.5 \mathrm{~A} / \mathrm{cm}$ if expressed in surface current units). Since the critical current should be at least less than these values, only the depinning onset close to the high temperature first order transition can be reliably measured. It is worth noting that similarly low current densities are usually not sufficient to 
overcome unavoidable critical current inhomogeneities at the onset of vortex depinning, even in pure superconducting metals. In this regime, only parts of the VL are moving. To reach the flux-flow regime where the whole $\mathrm{VL}$ is in motion, it is necessary to increase the value of the injected current, say $\left(I-I_{c}\right)$ reasonably larger than $I_{c}$. One of the possibilities which allows to overcome the overheating problems is the use of fast current pulses. This technique was employed in [8]. Both stable linear and metastable $\mathrm{V}(\mathrm{I})$ curves with a S-shape were measured. Since the pulse of current injection is very fast (about $100 \mu \mathrm{s}$ ), a normal skin effect can affect the preceding results and it is not completely clear if they can be taken as representative of the steady state of the moving VL. A confirmation of this experiment, using a continuous transport current, appears thus necessary. For that, we have studied monocrystalline bridges of 100 or $200 \mu \mathrm{m}$ of width. This ensures a good homogeneity of the current injection. Above all, for a same current I, a largest current density $(I / t W=J$ or $I / 2 W=i)$ is obtained when using a microbridge rather than a bulk sample. This let the possibility of working in the flux-flow regime without the overheating at the resistive contact pads, even at low temperature.

In this experiment, we would like to address simple experimental facts to the following questions:

- What are the fundamental differences, if any, in the shapes of the $\mathrm{V}(\mathrm{I})$ curves for the different VL states? Is it possible to explain the critical current values?

The samples used in this study are slightly $\mathrm{Pb}$ doped single crystals of the Bi-2212 family $\left(\mathrm{Bi}_{1.8} \mathrm{~Pb}_{0.2} \mathrm{Sr}_{2} \mathrm{CaC} u_{2} \mathrm{O}_{8+\delta}\right)$. They were grown by the self-flux technique as previously described [9]. Each cleaved single crystal was laser tailored in the form of a microbridge with a controlled pattern of $(\mathrm{W}=200 *$ $\mathrm{L}=400) \mu m^{2}$ or $(\mathrm{W}=100 * \mathrm{~L}=400) \mu m^{2}$ (Fig.2). The thickness is about $100 \mu \mathrm{m}$. The crystal was after annealed under a controlled pure oxygen gas flow and is in the slightly overdoped regime $\left(T_{c}=79.5 \mathrm{~K}\right)$. Low resistance electrical contacts $(\leq 1 \Omega)$ were made by bonding gold wires with silver epoxy. The DC transport measurements were performed using a standard four probe method (cryostat Quantum Design with a 9T horizontal coil, external current source Adret and nanovolmeter Keithley). The results presented here correspond to the microbridge with $\mathrm{W}=200 \mu m$, otherwise it will be specified in the text.

RESULTS IN THE LOW TEMPERATURE REGIME

We have first performed $\mathrm{V}(\mathrm{I})$ curves at low temperature $(\mathrm{T}=5 \mathrm{~K})$ in order to minimize the effect of the thermal fluctuations. Let us discuss the results for high magnetic field values $(1 \mathrm{~T} \geq \mathrm{B} \leq 9 \mathrm{~T}$, Fig. 3$)$. The $\mathrm{V}(\mathrm{I})$ curves are very similar to those observed for a conventional vortex lattice, as it can be measured in low $T_{c}$ metals or alloys. In particular, they present the usual form $V=R_{f f}\left(I-I_{c}\right)$ as soon as I is slightly higher than the critical current $I_{c}\left(R_{f f}\right.$ is the flux-flow resistance of the VL). There is no evidence of an ohmic regime at low applied current and the depinning is rather stiff. Furthermore, we have tried to compare the effects of a Field Cooling (FC) and of a Zero Field Cooling (ZFC), or of a FC under different cooling rates. Always the same dissipation has been measured in the time scale of our experiment. In particular, no aging effect is observed on the critical current. This is not in agreement with a glassy nature of the VL governing transport properties.

When the magnetic field is decreased, a different behavior is observed in a restricted region of the phase diagram $(0.05 T \leq B \leq 0.2 T)$. If the $\mathrm{VL}$ is prepared after a FC, the $\mathrm{V}(\mathrm{I})$ curve exhibits a S-shape with a high threshold current $I_{\text {high }}$ [10], but only for the first increase of the current. After this initial ramp, a reproducible $I_{c}<I_{\text {high }}$ is always measured (Fig. 4). This has been previously observed in the pulsed-current experiments, and this state with a "high threshold current" has been evidenced as a metastable state with a very long relaxation time [8, 11]. Our measurement using a dc current proves that the observation of this state is not due to the kind of stimulation used. This observation is also in agreement with the observed supercooling of a high critical current state using a time-resolved local induction measurement [2]. We observe a hierarchy in the accessible threshold currents. Depending on the exact preparation of the FC state (cooling rate, value of the initial field, rate of the current injection ramp), numerous threshold values are accessible between $I_{h i g h}$ and $I_{c}$. It is clear that in such a regime where it is easy to lock an out of equilibrium state, most of the measurements will give transient and spurious relaxation effects if the field is ramped or quenched as it is done in magnetization measurements. We find some traces of this metastability up to about $1 \mathrm{~T}$, but the most obvious effects are restricted in the region (0.05-0.2T) (Fig.5). For $I>I_{\text {high }}$, the $\mathrm{V}(\mathrm{I})$ curve is observed disrupted. If the width of the microbridge is narrower, multiple steps in the current induced moving state can even be observed (inset of fig. 4). Even small $(100 \mu \mathrm{m})$, this width is much larger than any superconducting length. It is thus likely that these structures in the $\mathrm{V}(\mathrm{I})$ curves can not be explained by phase slippage processes as observed in one dimensional superconductors [12]. This mimics rather the voltage steps which have been observed in the current-induced resistive state of type I superconductor 13] and can be the counterpart of a coexistence between two states possessing different critical currents and comparable spatial extensions. This dynamic is close to what is observed in a current carrying inhomogeneous superconductor. It can not be exclude that the high values of the threshold current are responsible for local heating in the interfaces between the domains and favor the formation of resistive "electrothermal" structures 14]. Concerning the metastable $\mathrm{V}(\mathrm{I})$ curves, the peak effect in the critical current, the coexistence of two VL states, the same kind of behavior is currently observed in $2 \mathrm{H}-\mathrm{NbSe}_{2}$ [15]. The strong difference is that the peak effect and the associated metastable effects appear close to $B_{c 2}$ in $\mathrm{NbSe}_{2}$ but is here restricted 
to a very low field value. Since the applied temperature is very similar in both experiments, the explanation of this field value difference has to be found in the large difference between the electronic anisotropies. For a field lower than about $0.05 \mathrm{~T}$, we do not observe any hysteresis within the $\mathrm{V}(\mathrm{I})$ curves. This is summarized in fig. 5 where $I_{c}$ versus $B$ is shown.

One has to remark that the variation of $I_{c}(B)$, if one excepts the small low field part where metastability takes place, looks like what is measured in soft low $\mathrm{T}_{c}$ materials. To some extent, one can speculate that the same pinning mechanism is acting without involving a different VL phase. Qualitatively, the functional form of $I_{c}(B)$ is very close to the one of the reversible magnetization of a high $\kappa$ anisotropic superconductor [17], meaning that $I_{c}$ is linked to the weight of the diamagnetic screening currents. This has to be brought close to the linear V(I) curves that we measured. We will return to this point later.

HIGH TEMPERATURE RESULTS: THE "LIQUID" STATE

Let us now discuss the $\mathrm{V}(\mathrm{I})$ curves obtained at high temperature. The temperature $\mathrm{T}$ was fixed at $50 \mathrm{~K}$ and the magnetic field $\mathrm{B}$ was varied from 0.001T to 9T, in order to cross the transition between the so-called "solid" and "liquid" vortex phases. In Bi-2212, thermodynamical consistency required by the respect of the ClausiusClapeyron relations [18] can not be proved, because up to now no transition can be detected using specific heat measurements. The small step usually observed in the magnetization [16], added to the reasonable idea that the same physics is acting both in $\mathrm{Bi}-2212$ and in $\mathrm{YBaCuO}$, can be taken as a good indication that this transition is of first order. The appearance of an approximate linear resistivity when the sample is probed at very low current density is also usually taken as a good criteria. The underlying ideas are that a liquid state of $2 \mathrm{D}$ vortices can not be pinned and that the resistive properties are close to that of a metal (Ohmic regime). Using the same criterion, the transition would be located at about $0.08 \mathrm{~T}$ (fig. 6), in agreement with values currently reported in the literature $(0.065 \mathrm{~T}$ in [16] for a Bi-2212 with an equivalent doping). Nevertheless, we observe that for magnetic fields largely higher, in a large part of what is identified as the "liquid" state, the V(I) curves are clearly non ohmic (fig. 7). A non linear response in this resistive state has already been observed. It has been interpreted as the feature of a highly viscous liquid of vortices 19], or by the edge effect of surface barriers (SB) [20]. Roughly speaking, SB are expected to be negligible for fields $B \geq B_{c 1} \kappa / \ln (\kappa) \approx 20 B_{c 1}$ for high $\kappa$ superconductor $(\kappa=100)$ with an ideal flat surface 21]. Any real sample also possesses surface irregularities which facilitate vortex nucleation and decrease this value. We estimate that, taken $B_{c 1} \leq 300 G$, SB effects can be neglected when a magnetic field of several Teslas is applied. We note also that other experiments performed using the Corbino geometry do not evidence any effect of
SB at high temperature 22]. We have also checked that a decrease of the sample width decreases $I_{c}$, confirming that neglecting SB is reasonable, at least as far as critical current properties at high field values are involved. We stress on the following points: the high current part of the $\mathrm{V}(\mathrm{I})$ curves is linear and its extrapolation never goes to zero (fig. 7), at least for $\mathrm{B} \leq 9 \mathrm{~T}$. In other terms, putting aside the rounded dissipation onset, $\mathrm{V}(\mathrm{I})$ curves can be expressed as $V=R_{f f}\left(I-I_{c}\right)$ as it was observed at low temperature. It can not be explained by a non linear mechanism in which a depinning energy would be a function of the driving force required to overcome barriers, because the high current regime is linear but does not reach an asymptotic Ohmic regime $(V=R . I)$. This demonstrates the existence of a real critical current $0 \leq I_{c} \leq 10 \mathrm{~mA}$ in this high temperature state. This critical current exhibits a field dependence that compares well with the one obtained at low temperature. It is thus reasonable to think that the same pinning mechanism occurs at low and high temperatures. The difference in the low dissipation level can be understandable in terms of an additive process which appears at high temperature, and a thermally activated process which assists the depinning is a natural candidate. This was largely studied in the literature [1]. This defines a threshold current I* above which a small dissipation appears but pinning continues to exist (Fig.6).

\section{DISCUSSION}

From a theoretical point of view, vortex lattice depinning is generally described like a critical phenomena: the bulk depinning of an elastic system in a random media. Driven states are described with an overdamped dynamical equation and it is expected that without thermal activation the velocity $v$ just above the depinning scales like $\left(F-F_{c}\right)^{\beta}$ where $F$ is the applied force, $F_{c}$ the bulk pinning force and $\beta$ is the critical exponent [1, 23]. The usual analysis supposes that $\left(F-F_{c}\right)^{\beta}$ identifies with $\left(J-J_{c}\right)^{\beta}, J$ being a current bulk density. At high velocity, disorder is found not relevant that leads to a velocity $v \alpha F$. Experimentally, this should correspond to an Ohmic regime. Clearly, the experimental results are different. Even without performing a detailed analysis, one can realize that the $\mathrm{V}(\mathrm{I})$ curves at the depinning onset are never observed convex. $\beta$ is never lower than 1 even at low temperature where the vortex Creep driven by thermal activation is negligible. One excludes from this discussion the peculiar case of the peak effect where a convex part is effectively observed but is very likely due to the macroscopic inhomogeneity of the state. It is more important to remark that, when $I \gg I_{c}$, the velocity is found to vary like $\left(I-I_{c}\right)$ and not like $I$. This means that the pinning force felt by the VL remains constant even at "high velocity", in apparent contradiction with an explanation in terms of a bulk depinning. One could object that the applied current in our experiment is not sufficiently high to reach the predicted Ohmic-like regime. This problem can never be strictly resolved because Joule heating is always a limitation when increasing the cur- 
rent in a transport experiment. Nevertheless, one can refer to low $\mathrm{T}_{c}$ materials where the experimental situation is much more attractive. Under very controlled temperature, it is possible to verify $V \alpha\left(I-I_{c}\right)$ up to at least $I \geq 30 I_{c}$. This is thus the generic shape of a $\mathrm{V}(\mathrm{I})$ curve in a type II superconductor, and the present experiment shows that the same dissipation mechanisms are acting in Bi-2212 samples. It has been also verified by inelastic neutron scattering that, in this regime, the VL is moving freely as a whole 24]. This latter result means that the bulk disorder can be estimated to be no more effective. At the same time, the former result shows that the velocity does not reach the asymptotic regime where the velocity should vary linearly with the total current. To be coherent with the above mentioned theories [23], one possible solution is to replace by hand the applied force $F(\operatorname{resp} I)$ that acts against bulk disorder by $\left(F-F_{c}\right)$ $\left(\operatorname{resp}\left(I-I_{c}\right)\right)$ (Fig. 9). Physically, this solution appears in the case of vortex pinning by the surface roughness [25]. The main idea is that the surface disorder (a quite standard surface roughness) allows, thanks to boundary conditions, for the flow of non dissipative superficial current $\left(i_{c}(A / \mathrm{cm})=I_{c} / 2 W\right)$. This is only when all the superficial non dissipative paths are exhausted, precisely when $I \geq I_{c}$, that the excess of current $\left(I-I_{c}\right)$ penetrates the bulk. To some extent, a bulk force makes then sense and a "bulk depinning" can occurs, but involving only the over critical part of the applied current. The bulk disorder can be averaged by the motion without affecting the main critical current which reflects the surface pinning ability, explaining the experimental shape of the $\mathrm{V}(\mathrm{I})$ curves. As an further indication that this mechanism can occur in the Bi-2212 samples, quantitative expressions for $I_{c}(B)$ are predicted and can be checked. The case of very anisotropic samples is specially interesting, because it is predicted that for not too low magnetic field values and for a moderate surface roughness, the surface critical current $i_{c}$ may become independent of the surface quality and depends then solely on the parameters of the condensate [17]. For clarity, we restrict the comparison to the high field values in order to use the Abrikosov limiting expressions [17]:

$$
i_{c}=\frac{B_{c 2}}{2 \mu_{0} \beta_{A} \gamma \kappa_{2 / / c}(T)^{2}}\left(1-\left(B / B_{c 2}\right)^{2 / 3}\right)^{3 / 2}
$$

Here, $\kappa_{2 / / c}(T)$ is the generalized Ginzburg-Landau parameter, $\gamma$ the electronic anisotropy and $B_{c 2}$ the second critical field. For the small temperature dependence of $\kappa_{2}$, we use the microscopic GL derivation [26]. This expression describes rather well the data (Fig.10), with the use of a restricted number of parameters. $\gamma=60$ was obtained from angular resolved resistivity measurements using scaling arguments [27]. With $\kappa_{2}=\frac{\lambda}{\xi}, B_{c 2}=\frac{\phi_{0}}{2 \pi \xi^{2}}$, we find $\xi_{(0)}=3.3 \mathrm{~nm}, \lambda_{(0)}=2080 \mathrm{~nm}$ consistent with the slightly overdoped regime 28]). The approximate field dependence of the critical current in a restricted field range is an indication but can not be taken as a proof of the validity of the model. Other field dependence would be acceptable. In particular, in a log-log representation, it is reasonable to estimate that $I_{c}$ varies like $B^{-0.5}$, as it is often observed ([8] and references herein) and interpreted as a $2 \mathrm{D}$ strong bulk pinning regime. A possible limitation of this latter analysis is the relevance of the strong bulk pinning centers in clean single crystals. It is more important to note that the absolute value of the critical current is the one expected if only the surface contributes to pinning. To compare with other experiments, we measured $i_{c} \approx 0.1-0.5 \mathrm{~A} / \mathrm{cm}$ at $(\mathrm{T}=5 \mathrm{~K}, \mathrm{~B}=$ 9 to $1 \mathrm{~T}$ ) in our microbridge, and $0.4-1 \mathrm{~A} / \mathrm{cm}$ was measured in [8]. These values seem representative of Bi-2212 crystals. Now it can be objected that the current distribution in Bi-2212 can be strongly influenced by the very large electronic anisotropy [32]. This leads to a very small current penetration in the normal and in the superconducting state and this has a priori no link with any pinning mechanism. It remains that if a current flowing under the surface is non dissipative up to a critical value, a surface pinning mechanism should apply. Finally, it seems that there is no need to involve bulk disorder in order to explain the critical current and the shape of the $\mathrm{V}(\mathrm{I})$ curves at least outside the peak effect region. Other experiments will soon be performed to evaluate more precisely the validity of this hypothesis and the specific case of the peak effect will be discussed in another paper.

It is clear that this interpretation of the $\mathrm{VL}$ pinning in Bi-2212 is at odds with the currently accepted view that the pinning is driven by the bulk disorder (collective bulk pinning). We need to add some comments to replace this result in the context of recent experiments.

In Bi-2212, the peak effect should separate a high field disordered phase with glassy properties the low field ordered VL. The VL, corresponding to the V(I) curves of the fig.3, is expected to be in this glassy state, whereas we observe a conventional VL dynamic rather than a glassy dynamic in its proper sense. The experimental proof of a disordered high field state in Bi-2212 is also not completely obvious. Small Angle Neutron Scattering experiments can effectively be interpreted in terms of a VL disordering transition at very low field [29]. This interpretation relies in the strong decrease of the diffracted intensity when the density of vortices increases, whereas a simple London model which disregards the finite size of the vortex core predicts only a smooth decrease due to geometrical factors. Nevertheless, recent theoretical progresses allow to calculate the microscopic field distribution and its Fourier components are found to be strongly field dependent even at low field (for high kappa and/or anisotropic superconductors) [30], meaning that involving a transition in the VL may be not necessary. This can explain why other experiments with a largest intensity are showing that a well ordered VL with good Bragg peaks survives at higher fields [31]. We note also that recent experiments suggest that the VL order is not relevant for the nature of the "melting" transition in Bi-2212 [4], meaning that the order parameter of this transition 
is not linked to the topology of the VL. Experimental data suggest that the line of the peak effect in the phase diagram is the continuation of the first order line into the low-temperature [16]. This implies that the same state of the VL is present at low and high temperature. Our experiment suggests that the pinning of the vortex lattice is also of the same nature, even if thermal fluctuations are responsible for a low dissipation background at high temperature. Finally, it has been recently proposed that the renormalization of the (non-local) line tension by thermal fluctuations for large wave vectors is at the origin of the first order transition [33]. As the line tension is the controlling parameter for the surface pinning, this offers a scenario more compatible with our results than the occurrence of a genuine melting transition. Furthermore, even if the bulk condensate is strongly fluctuating with eventually disrupted vortices, a small dissipationless current can persist under the surfaces. A well known example is the case of surface superconductivity where a critical current does exist in the surface sheath even if the bulk develops into its normal state.

To conclude, the use of monocrystalline microbridges of Bi-2212 allowed us to measure V(I) curves in different parts of the VL phase diagram. They appear conventional and a clear critical current can be defined by extrapolating the high current linear part. A metastable high threshold state can be stabilized by Field Cooling. This corresponds to the peak effect region and mimics what is observed much closer to $B_{C 2}$ in $\mathrm{NbSe}_{2}$. The values of the measured critical currents and the shapes of the $\mathrm{V}(\mathrm{I})$ curves suggest that the pinning by the surface roughness can not be ignored for understanding the vortex lattice dynamic in Bi-2212. We observe that this superficial critical current survives in a large part of the so-called liquid state. Finally, the overall picture suggests that the VL pinning and dynamic in $\mathrm{Bi}-2212$ are more similar to what is currently observed in low $T_{c}$ materials than it is often suggested.

Acknowledgments: Joseph Scola acknowledges support from "la région basse Normandie".
[1] T. Giamarchi , S. Bhattacharya, Vortex Phases in "High Magnetic Fields: Applications in Condensed Matter Physics and Spectroscopy", C. Berthier et al., 9, 314, Springer-Verlag (2002).

[2] C.J. van der Beek, S. Colson, M.V. Indenbom, M. Konczykowski, Phys. Rev. Lett. 84 (2000).

[3] N. Avraham, B. Khaykovich, Y. Myasoedov, M. Rappaport, H.Shtrikman, D.E. Feldman, T. Tamegai, P. Kes, M. Li, M. Konczykowski,K. van der Beek, and E. Zeldov, Nature (London) 411, 451 (2001).

[4] M.Menghini, Y.Fasano, F.de la Cruz, S.S.Banerjee, Y.Myasoedov, E.Zeldov, C.J.van der Beek, M.Konczykowski and T.Tamegai, Phys. Rev. Lett. 90, 147001 (2003).

[5] M. Cristina Marchetti, A. Alan Middleton, and Thomas Prellberg, Phys. Rev. Lett. 85, 1104 (2000).

[6] T. Giamarchi and P. Le Doussal, Phys. Rev. Lett. 76, 3408 (1996).

[7] C. J. Olson, C. Reichhardt, and V. M. Vinokur, Phys. Rev. B 64, 140502 (2001).

[8] B. Sas, F. Portier, K. Vad, B. Keszei, L. F. Kiss, N. Hegman, I. Puha, S. Mszros, and F. I. B. Williams, Phys. Rev. B 61, 9118 (2000).

[9] A.Ruyter, Ch.Simon, V.Hardy, M.Hervieu, A.Maignan, Physica C 225, 235 (1994).

[10] Since $I_{h i g h}$ is not reflecting the equilibrium state, we use the term threshold current, and we limit the use of the term critical current for the depinning current which is not history dependent.

[11] F. Portier, G. Kriza, B. Sas, L. F. Kiss, I. Pethes, K. Vad, B. Keszei, and F. I. B. Williams Phys. Rev. B 66, 140511 (2002).

[12] W. J. Skocpol, M. R. Beasley, and M. Tinkham, J. Low Temp. Phys. 16, 145 (1974).

[13] R. P. Huebener and H. L. Watson, Phys. Rev. B 9, 37253729 (1974).

[14] A.VI. Gurevich and R.G. Mints, Rev. Mod. Phys. 59, 941 (1987).
[15] M.J. Higgins and S. Bhattacharya, Physica C 257, 232 (1996).

[16] B. Khaykovich, E. Zeldov, D. Majer, T. W. Li, P. H. Kes, and M. Konczykowski, Phys. Rev. Lett. 76, 2555 (1996).

[17] G. Lazard, P. Mathieu, B. Plaçais, J. Mosqueira, Y. Simon, C. Guilpin, and G. Vacquier, Phys. Rev. B 65, 064518 (2002).

[18] F. Bouquet, C. Marcenat, E. Steep, R. Calemczuk, W.K Kwok, U. Welp, G.W. Crabtree, R.A. Fisher, N.E. Phillips, A. Schilling, Nature 411, 448 (2001).

[19] T. Tsuboi, T. Hanaguri, and A. Maeda, Phys. Rev. B 55, R8709 (1997).

[20] D. T. Fuchs, E. Zeldov, T. Tamegai, S. Ooi, M. Rappaport, and H. Shtrikman, Phys. Rev. Lett. 80, 4971 (1998).

[21] L. Burlachkov, A. E. Koshelev and V. M. Vinokur, Phys. Rev. B 54, 6750 (1996).

[22] A. Mazilu, H. Safar, D. Lpez, W. K. Kwok, G. W. Crabtree, P. Guptasarma, and D. G. Hinks, Phys. Rev. B 58, R8913-R8916 (1998).

[23] T. Nattermann, S. Scheidl, Adv. Phys. 49, 607 (2000). P. Chauve, T. Giamarchi, P. Le Doussal, Phys Rev B 62, 6241 (2000). M. Mueller, D. Gorokhov, G. Blatter, Phys. Rev. B 63, 184305 (2001).

[24] E. M. Forgan, P. G. Kealey, S. T. Johnson, A. Pautrat, Ch. Simon, S. L. Lee, C. M. Aegerter, R. Cubitt, B. Farago, and P. Schleger Phys. Rev. Lett. 85, 3488 (2000).

[25] T. Hocquet, P. Mathieu, and Y. Simon, Phys. Rev. B 46, 1061 (1992).

[26] E. Helfand and N. R. Werthamer, Phys Rev. 147, 288 (1966).

[27] H. Raffy, S. Labdi, O. Laborde, and P. Monceau, Phys. Rev. Lett. 66, 2515 (1991).

[28] G. Villard, D. Pelloquin, and A. Maignan, Phys. Rev. B 58, 15231 (1998).

[29] R. Cubitt, E. M. Forgan, G. Yang, S. L. Lee, D. McK. Paul, H. A. Mook, M. Yethiraj, P. H. Kes, T. W. Li, A. A. Menovsky, Z. Tarnawski and K. Mortensen, Nature 365, 407 (1993). 
[30] E.H. Brandt, Phys. Rev. Lett. 78, 2208 (1997).

[31] E. M. Forgan, M. T. Wylie, S. Lloyd, S. L. Lee, and R. Cubitt, Proc. LT-21: Czechoslovak Journal of Physics 46 1571 (1996).

[32] I. Pethes, A. Pomar, B. Sas, G. Kriza, K. Vad, A. Pallinger, F. Portier, and F. I. B. Williams, Phys. Rev.
B 68, 184509 (2003).

[33] S.Colson, M.Konczykowski, M.B.Gaifullin, Y.Matsuda, P.Gierlowski, M.Li, P.H.Kes, and C.J.van der Beek, Phys.Rev.Lett. 90, 137002 (2003). 


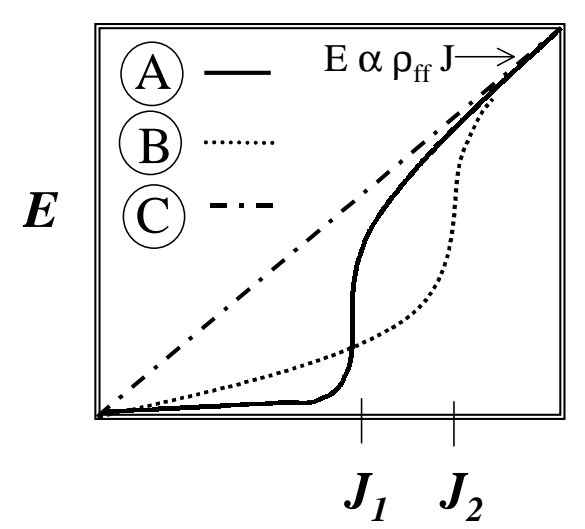

FIG. 1: V(I) curves expected for different phases of the VL (A:Quasi-Lattice (Bragg Glass), B:Glass, C:Liquid). $J_{1}$ represents the depinning current in the phase $\mathrm{A}, J_{2}$ in the phase $\mathrm{B}$.

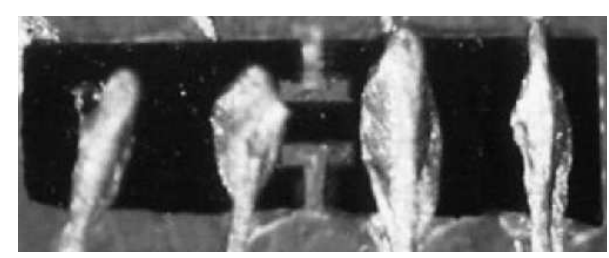

FIG. 2: The monocrystalline microbridge $\left(200 * 400 \mu m^{2}\right)$ of Bi-2212.

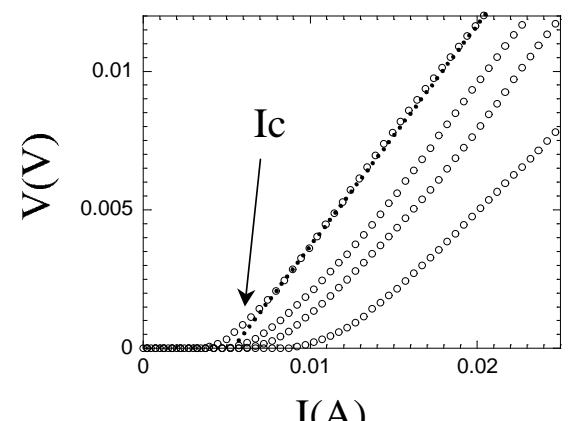

I(A)

FIG. 3: Reversible $\mathrm{V}(\mathrm{I})$ curves $(\mathrm{T}=5 \mathrm{~K}, \mathrm{~B}=1,3,5,9 \mathrm{~T}$ from the right to the left). The dashed and straight line is a guide for the eyes and evidences the flux - flow regime.

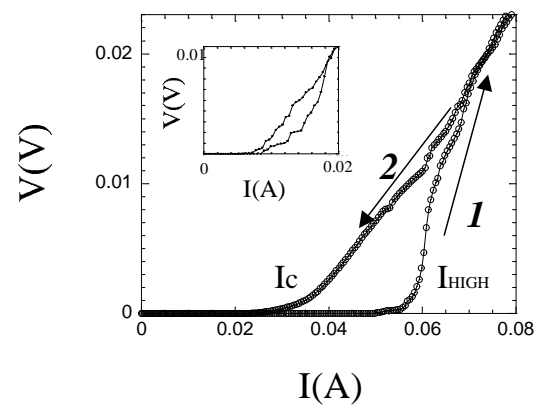

FIG. 4: Hysteretic $\mathrm{V}(\mathrm{I})$ curve $\left(\mathrm{T}=5 \mathrm{~K}, \mathrm{~B}=0.15 \mathrm{~T}\right.$ after a FC).1/ The first increase of current defines $I_{h i g h}$ and $2 /$ The following decrease of the current defines $I_{c}$. In the inset is shown the same kind of curve in the microbridge with a smallest width $(\mathrm{T}=5 \mathrm{~K}, \mathrm{~B}=0.05 \mathrm{~T})$. Note the steps in the $\mathrm{V}(\mathrm{I})$ curve. 


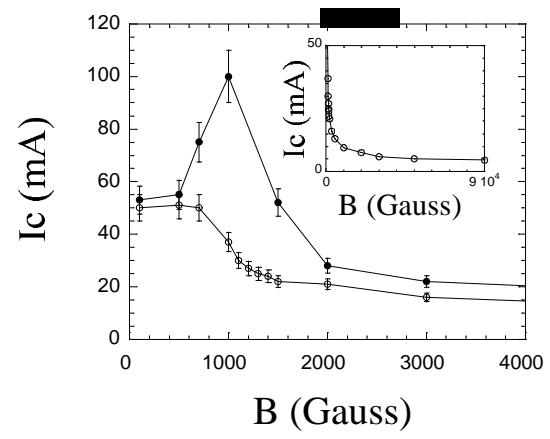

FIG. 5: Peak effect in the critical current $\left(\mathrm{T}=5 \mathrm{~K}\right.$. Black points, $I_{h i g h}$ and empty points, $\left.I_{c}\right)$. In the inset is shown the variation of $I_{c}$ at high field.

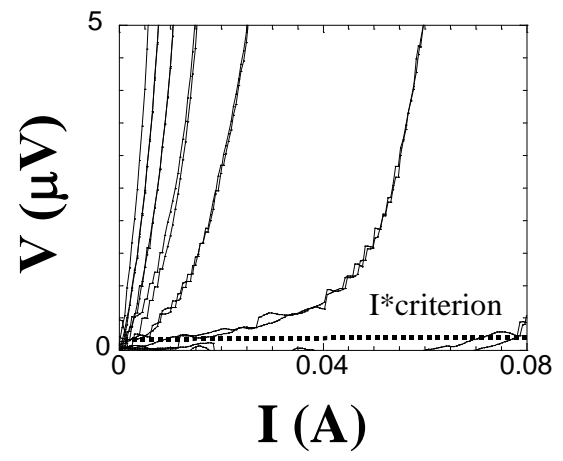

FIG. 6: Low dissipation level of the $\mathrm{V}(\mathrm{I})$ curves $(\mathrm{T}=50 \mathrm{~K}$, from the left to the right: $\mathrm{B}=0.14 \mathrm{~T}, 0.12 \mathrm{~T}, 0.1 \mathrm{~T}, 0.08 \mathrm{~T}, 0.06 \mathrm{~T}$, $0.04 \mathrm{~T}, 0.02 \mathrm{~T})$. As shown in the figure, $I_{c}^{*}$ is defined using a criterion of $0.2 \mu V$ and is nearly zero for $\mathrm{B} \gtrsim 0.08 \mathrm{~T}$.

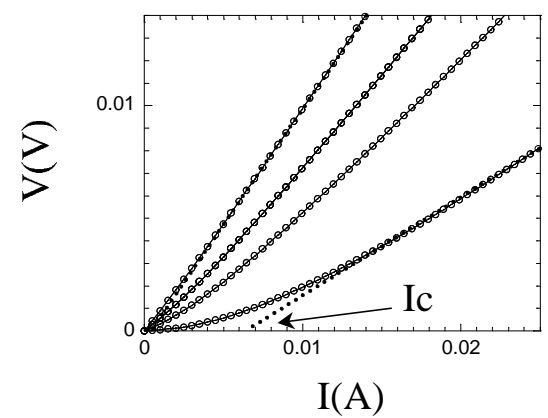

FIG. 7: Reversible $\mathrm{V}(\mathrm{I})$ curves ( $\mathrm{T}=50 \mathrm{~K}, \mathrm{~B}=1,3,5,9 \mathrm{~T}$ from the right to the left). The dashed and straight line is a guide for the eyes. This linear extrapolation defines $I_{c}$. Note that the regime is clearly non ohmic up to at least $\mathrm{B}=9 \mathrm{~T}$. 


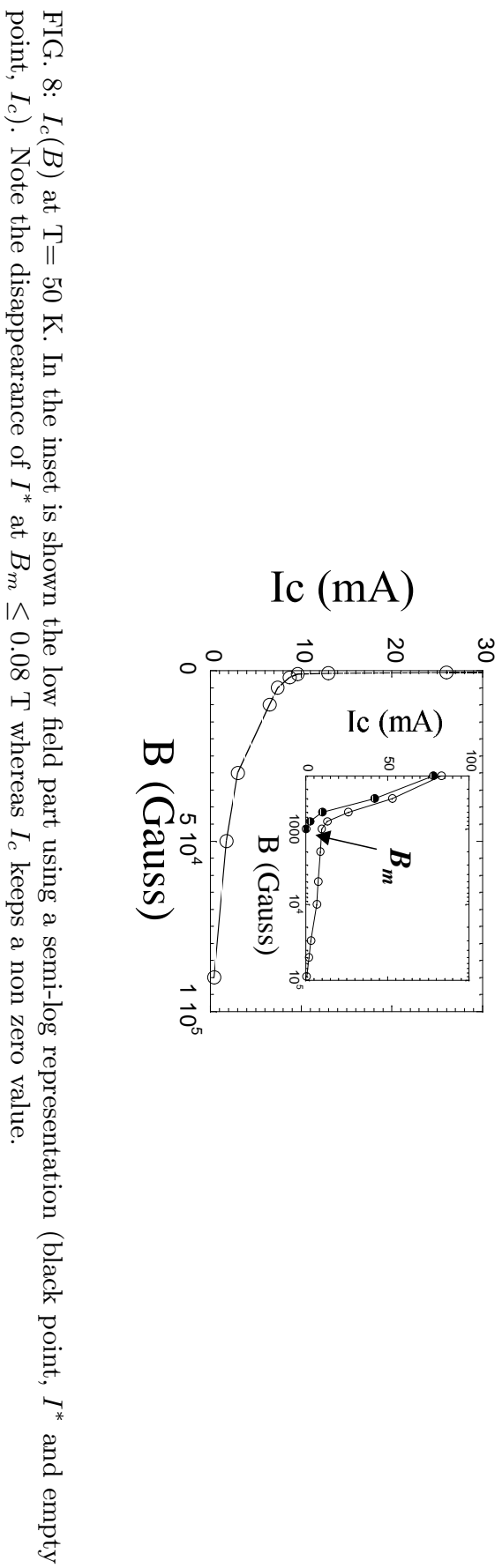

